\title{
SOME POSSIBILITIES FOR CHARM STUDIES AT B-FACTORIES
}

\author{
E. GOLOWICH \\ Physics Department, University of Massachusetts, Amherst, \\ MA 01003, USA
}

\begin{abstract}
To provide focus to my review of charm theory at the B-Physics and CP-Violation conference, I consider theoretical issues related to possible charm studies at $B$ factories. A few topics of interest to the author are stressed - rare decays, CPviolating asymmetries and $\bar{D}^{0}-D^{0}$ mixing.
\end{abstract}

\section{Introduction}

To me, the CKM matrix is first and foremost an object of phenomenological study. When I look at it, it is natural to associate with each matrix element at least one experiment, as in

$$
\left[\begin{array}{lll}
V_{\mathrm{ud}} & V_{\mathrm{us}} & V_{\mathrm{ub}} \\
V_{\mathrm{cd}} & V_{\mathrm{cs}} & V_{\mathrm{cb}} \\
V_{\mathrm{td}} & V_{\mathrm{ts}} & V_{\mathrm{tb}}
\end{array}\right] \leftrightarrow\left[\begin{array}{lll}
\beta \text { decay } & K \rightarrow \pi \ell \bar{\nu} & B_{d} \rightarrow X_{\mathrm{u}} \ell \bar{\nu} \\
\nu N \rightarrow \mu \bar{\mu} X & D \rightarrow \bar{K} \ell \bar{\nu} & B \rightarrow D^{*} \ell \bar{\nu} \\
B_{d}-\bar{B}_{d} & b \rightarrow s \gamma & t \rightarrow b W
\end{array}\right]
$$

The gradual and painstaking determination of the CKM matrix over a long period of time is a remarkable accomplishment of our field, and hopefully it will attain a level of completion from the B-factory studies to come.

The emphasis of $B$-factories will be on the physics of $b$ quarks and deservedly so. Already, there is discovery of a $\Delta B=2$ process $\left(\bar{B}_{d}-B_{d}\right.$ mixing $)$ and an EW-penguin decay $\left(B \rightarrow K^{*} \gamma\right)$. The future observation of CP violations (CPV) in $B$ decays would top the list.

However, $B$ factories will provide opportunities for doing $c$ quark studies as well. I would hope that the following items be given some consideration in the planning now underway:

- Search for flavor-changing neutral current (FCNC) charm decays, especially

$$
\begin{aligned}
& D \rightarrow M \gamma(M \text { denotes a } J>0 \text { non-charm meson }), \\
& D \rightarrow M^{\prime} \ell^{+} \ell^{-}\left(M^{\prime} \text { denotes a non-charm meson }\right),
\end{aligned}
$$

- Tighten the limit on CPV asymmetries in $D^{ \pm}$decay,

- Tighten the limit on $D^{0}-\bar{D}^{0}$ mixing.

Although many more possible studies of charm systems come to mind, this talk will concentrate on just these few. 
Table 1: Measured Phases.

\begin{tabular}{c|c} 
Mode & $\delta_{I}-\delta_{I^{\prime}}$ \\
\hline \hline$K \bar{K}$ & $\left(28_{-17}^{+9}\right)^{o}$ \\
$K \pi$ & $\left(97_{-13}^{+12}\right)^{o}$ \\
$K^{*} \pi$ & $(90 \pm 16)^{o}$ \\
$K \rho$ & $(10 \pm 47)^{o}$ \\
$K^{*} \rho$ & $(33 \pm 57)^{o}$ \\
$\pi \pi$ & $(81 \pm 10)^{o}$ \\
\hline \hline
\end{tabular}

\section{Current Status of Weak Decay Studies}

I shall begin by giving a very brief overview of the current status of the electroweak sector of charm physics. An important 'truth' evidenced in weak decays of charm is the relative importance of the strong interactions. This is already signalled by the large number of identifiable hadron resonances in the charm region. For example, in the most recent listing of the Particle Data Group, I count twelve non-strange and six strange resonances in the $1700 \rightarrow 2000 \mathrm{MeV}$ mass region. 1

\subsection{Experimental Studies}

If QCD effects are significant in the charm region, one expects phases to be prevalent in weak decay amplitudes due to effects of final state interactions (FSI). It is a sign of the maturity in experimental studies that such phases are routinely extracted from study of isospin sum rules. Table 1 summarizes the current state of affairs. 2 . An example is the recent CLEO analysis of the $D \rightarrow K \bar{K}$ system 2 in which the physical decay amplitudes are expressed in terms of isospin,

$$
A_{+-}=\frac{A_{1}+A_{0}}{\sqrt{2}}, \quad A_{+0}=\sqrt{2} A_{1}, \quad A_{00}=\frac{A_{1}-A_{0}}{\sqrt{2}} .
$$

This allows extraction of the phase difference $\delta_{\bar{K} K} \equiv \delta_{I=1}-\delta_{I=0}$ from the measured decay widths,

$$
\cos \delta_{\bar{K} K}=\frac{\Gamma_{+-}-\Gamma_{00}}{\left[\Gamma_{+0}\left(2 \Gamma_{+-}+2 \Gamma_{00}-\Gamma_{+0}\right)\right]^{1 / 2}}
$$


Table 2: DCSD Signals in the $K \pi$ Sector.

\begin{tabular}{l|c} 
Experiment & $r_{K \pi}^{\mathrm{DCSD}}$ \\
\hline \hline CLEO & $(0.77 \pm 0.25 \pm 0.25) \%$ \\
E791 & $\left(0.68_{-0.33}^{+0.34} \pm 0.07\right) \%$ \\
\hline \hline
\end{tabular}

\subsection{Theoretical Studies}

The list of theory papers on charm decays is very long. A recent and comprehensive study of $D$ decays is that of Buccella, Lusignoli and Pugliese, 1 who study a large number of modes and, most notably, include FSI effects in their fits. As a result, each of their weak decay amplitudes has a phase. This provides them with the ability to estimate both CPV asymmetries and $\Delta \Gamma_{D} / \Gamma_{D}$. We shall consider each of these in due course.

\section{Current Status of Exotic Physics Studies}

Suffice it to say that, despite a number of experimental searches, no truly exotic signals have yet been discovered in the charm sector. In the following, we shall touch on both the experimental and theoretical situations and indicate that at least some theoretical predictions are not terribly far from existing limits.

\subsection{Experimental Studies}

The only signal observed in this category is the detection of doubly-Cabibbosuppressed decay (DCSD), and one is clearly stretching things by calling this unambiguous SM phenomenon 'exotic'. A number of searches for FCNC processes have been carried out, but with null results. We shall list a few examples to indicate the present level of sensitivity.

\section{Observation of Doubly-Cabibbo-Suppressed Decay}

Although not truly exotic, DCSD occurs with a sufficiently small branching ratio to qualify as an impressive experimental achievement. We recall that the context for making the measurement is one where DCSD is a background to the more interesting possibility of $D^{0}-\bar{D}^{0}$ mixing. Results of mixing studies are usually cast in terms of the $r$-parameter, where

$$
r_{f}^{\operatorname{mix}} \equiv \frac{B_{D^{0} \rightarrow \bar{D}^{0} \rightarrow \bar{f}}}{B_{D^{0} \rightarrow f}}, \quad r_{f}^{\mathrm{DCSD}} \equiv \frac{B_{D^{0} \rightarrow \bar{f}}^{\mathrm{DCSD}}}{B_{D^{0} \rightarrow f}} .
$$


Table 3: Some Limits on FCNC Decays.

\begin{tabular}{c|c|c} 
Mode & Branching Ratio & Experiment \\
\hline \hline$D^{+} \rightarrow \pi^{+} \mu^{+} \mu^{-}$ & $<1.8 \times 10^{-5}$ & E791县 \\
$D^{0} \rightarrow \mu^{+} \mu^{-}$ & $<4.2 \times 10^{-6}$ & E771 1 \\
$D^{0,+} \rightarrow X^{0,+} \ell^{+} \ell^{-}$ & $<\mathcal{O}\left(10^{-4} \rightarrow 10^{-5}\right)$ & CLEO 1 \\
\hline \hline
\end{tabular}

In both CLEO 0 and E791日 experiments, the observed wrong-sign $K \pi$ signals were interpreted as DCSD events and the values displayed in Table 2 are seen to be consistent within error bars.

DCSP has also been observed in the $K 2 \pi$ sector $D^{+} \rightarrow K^{+} \pi^{-} \pi^{+}$mode by E687 and by E791 8 . In each case, a branching fraction $B_{D^{+} \rightarrow K^{+} \pi^{-} \pi^{+}} \simeq$ $3 \times \tan ^{4} \theta$ is observed. A Dalitz plot analysis of the E791 data reveals roughly equal amounts of $K^{+} \rho^{0}, K^{* 0} \pi^{+}$and nonresonant behavior.

\section{Searches for FCNC Decays}

A survey of searches for exotic decays appears elsewhere in these proceedings. We simply take note in Table 3 that the overall level of sensitivity has in some cases reached the $\mathcal{O}\left(10^{-6}\right)$ level.

\subsection{Theoretical Studies of FCNC Processes}

Let us suppose that the studies of $D \rightarrow M \gamma$ and/or $D \rightarrow M^{\prime} \ell^{+} \ell^{-}$recommended earlier in this talk are actually carried out and that some signal is observed. Several interpretations are possible:

- A signal of 'new physics', 12

- A SM short-distance effect, akin to the EW-penguin of $B \rightarrow K^{*} \gamma$,

- A SM long-distance effect.13

In the following, we explore this important issue with reference to the $D \rightarrow M \gamma$ and $D \rightarrow M^{\prime} \ell^{+} \ell^{-}$transitions. 


\section{Weak Radiative Decay $(D \rightarrow M \gamma)$}

The current experimental limit is $B_{D^{0} \rightarrow \rho^{0}, \omega^{0} \gamma}=\mathcal{O}\left(10^{-4}\right)$. Let us compare this with theoretical expectations. The charm analog of the short-distance EW penguin vertex $b \rightarrow s \gamma$ is $c \rightarrow u \gamma$. Omitting QCD corrections, one finds a tiny branching ratio $B_{c \rightarrow u \gamma}^{\mathrm{No} Q \mathrm{QD}} \simeq 1.4 \times 10^{-17}$. Although a study of one-loop QCD corrections 14 finds a large enhancement, the branching ratio $B_{c \rightarrow u \gamma}^{1-\text { loop }} \simeq 5 \times 10^{-12}$ is still tiny. The same is true even when two-loop QCD corrections are included 15 . However, two studies of long distance effects (using respectively vector-dominance arguments 14 and a weak-annihilation/QCDsum-rule approach 16 ) obtain branching ratios at the $\mathcal{O}\left(10^{-6}\right)$ level. One must therefore keep in mind that misinterpretation of observed FCNC signals is possible unless long range effects are first understood!

\section{Weak Decay into Lepton Pairs $\left(D \rightarrow M^{\prime} \ell^{+} \ell^{-}\right)$}

The process $D \rightarrow \pi \ell^{+} \ell^{-}$is analogous to the weak radiative decay just considered except that the photon is now virtual (we denote a virtual photon as $\gamma^{*}$ ) and must carry sufficient momentum transfer to produce an on-shell lepton pair. The branching ratio from the short-distance (EW penguin) vertex $c \rightarrow u \gamma^{*}$ in the literature is $\mathcal{B}_{D \rightarrow \pi \ell^{+} \ell^{-}}^{\mathrm{EW}}=\mathcal{O}\left(10^{-8}\right)$. However, there is an almost trivial source of lepton pairs arising from the decay chain

$$
D \rightarrow \pi+\phi \rightarrow \pi+\ell^{+} \ell^{-} .
$$

Using as input the measured branching ratio $B_{D^{+} \rightarrow \pi^{+} \phi^{0}}=(6.1 \pm 0.6) \times 10^{-3}$, it is estimated from integrating $d \Gamma_{D^{+} \rightarrow \pi^{+} \ell^{+} \ell^{-}} / d m_{\ell^{+} \ell^{-}}^{2}$ over the $\phi$ peak that a 'resonance' branching ratio $B_{D^{+} \rightarrow \pi^{+} \phi^{0}}^{\text {res }} \simeq 0.8 \times 10^{-6}$ occurs and that the wings of the Breit-Wigner profile of the $\phi$ lead to branching ratios $\mathcal{O}\left(10^{-7}\right)$, the precise magnitudes of which depend on cuts in $d \Gamma_{D^{+} \rightarrow \pi^{+} \ell^{+} \ell^{-}} / d m_{\ell^{+} \ell^{-}}^{2}$. 17

\section{CP Violation in the $D$ System}

Although there is not time to fully review this subject, I consider two matters of special interest and worthy of comment.

\subsection{Time Dependence in $\bar{D}^{0}-D^{0}$ Mixing}

An approximate but accurate formula for thetime-dependent decay rate for the transition of $D^{0}$ to some final state $f$ is 18.29

$$
\Gamma_{D^{0}(t) \rightarrow f} \propto e^{-\Gamma_{D} t}\left[X+Y t+Z t^{2}\right],
$$


Table 4: Mixing Signals.

\begin{tabular}{l|c} 
Mixing & $r_{K \pi, K 3 \pi}^{\operatorname{mix}}$ \\
\hline \hline $\bar{D}^{0} \rightarrow D^{0}$ & $\left(0.18_{-0.39}^{+0.43} \pm 0.17\right) \%$ \\
$D^{0} \rightarrow \bar{D}^{0}$ & $\left(0.70_{-0.53}^{+0.58} \pm 0.18\right) \%$ \\
\hline \hline
\end{tabular}

where $x_{D}, y_{D} \ll 1$ and $|\lambda| \ll 1$ ( $\lambda$ is defined below). The constant term $X$ within the brackets arises from DCSD, whereas the quadratic term $Z$ is entirely due to mixing. The linear term $Y$ arises from interference and can be written as

$$
Y=2 \mathcal{R} e \lambda \Delta \Gamma_{D}+4 \mathcal{I} m \lambda \Delta m_{D},
$$

where $\lambda$ is a complex number defined by $\lambda \equiv \frac{p}{q} \frac{A}{B}$ with $p$ and $q$ being the usual mass matrix parameters and

$$
A \equiv\left\langle f\left|H_{\mathrm{wk}}\right| D^{0}\right\rangle, \quad B \equiv\left\langle f\left|H_{\mathrm{wk}}\right| \bar{D}^{0}\right\rangle .
$$

If one neglects the presumably small $\Delta \Gamma_{D}$ contribution to the $Y$ term, the remaining contribution is proportional to $\mathcal{I} m \lambda$, which can be nonzero if (i) CPV is present (thus inducing a phase in $p / q$ ) and/or (ii) the FSI are different in $D^{0} \rightarrow f$ and $\bar{D}^{0} \rightarrow f$ (thus inducing a phase in $A / B$ ). Blaylock et al performed a careful analysis of the time dependence in mixing and discussed the importance of allowing for FSI effects in any model-independent experimental analysis. 19 Table 1 displays the mixing values obtained from nonleptonic decays $(D \rightarrow K \pi$ and $D \rightarrow K 3 \pi)$ and obtained under the most general of conditions, i.e. making no assumptions regarding the absence of CPV. The $90 \%$ C.L. upper bounds are $r_{K \pi, K 3 \pi}^{\operatorname{mix}}\left(\bar{D}^{0} \rightarrow D^{0}\right)<0.74 \%$ and $r_{K \pi, K 3 \pi}^{\operatorname{mix}}\left(D^{0} \rightarrow \bar{D}^{0}\right)<1.45 \%$. It is striking that despite the very large data sample of E791, the resulting bound on $D^{0}-\bar{D}^{0}$ mixing is of the same order-of-magnitude as previously determined. Part of the reason for this is the careful attention paid by E791 to the possibility of an interference term.

\subsection{CP Violating Asymmetries in $D^{ \pm}$Decay}

In meson systems, one can seek CPV effects either via mixing (indirect CPV) or not (direct CPV). For $D$ mesons, it would appear that the former can be observed only from some kind of new physics mechanism. For the purpose of testing CPV in the Standard Model, the search for asymmetries in $D^{ \pm}$decays is particularly important. The E791 results given in Table 5 reveal that the current sensitivity of such searches is in the $\mathcal{O}\left(10^{-2}\right)$ range. 
Table 5: E791 Limits on CPV Asymmetries.

\begin{tabular}{c|c|c} 
Mode & $a_{\mathrm{CP}}$ & $90 \%$ CL Limits $(\%)$ \\
\hline \hline$K^{-} K^{+} \pi^{+}$ & $-0.014 \pm 0.029$ & $-6.2<a_{\mathrm{CP}}<3.4$ \\
$\phi \pi^{+}$ & $-0.028 \pm 0.036$ & $-8.7<a_{\mathrm{CP}}<3.1$ \\
$\bar{K}^{* 0}(892) K^{+}$ & $-0.010 \pm 0.050$ & $-9.2<a_{\mathrm{CP}}<7.2$ \\
$\pi^{-} \pi^{+} \pi^{+}$ & $-0.017 \pm 0.042$ & $-8.6<a_{\mathrm{CP}}<5.2$ \\
\hline \hline
\end{tabular}

As regards theoretical predictions, it has been suggested that the $\rho \pi$ mode would be particularly attractive,

$$
a_{\mathrm{CP}}^{(\rho \pi)} \equiv \frac{\Gamma_{D^{+} \rightarrow \rho^{0} \pi^{+}}-\Gamma_{D^{-} \rightarrow \rho^{0} \pi^{-}}}{\Gamma_{D^{+} \rightarrow \rho^{0} \pi^{+}}+\Gamma_{D^{-} \rightarrow \rho^{0} \pi^{-}}} \simeq-2 \times 10^{-3} .
$$

More generally, this reference predicts a number of CPV asymmetries at the $\mathcal{O}\left(10^{-3}\right)$ level. This is curiouly near the maximum expected from the SM as estimated out by Burdman,20

$$
\begin{aligned}
\left|a_{\mathrm{CP}}^{\text {(charm) }}\right| & \sim \frac{\mathcal{I} m\left[V_{\mathrm{cd}} V_{\mathrm{ud}}^{*} V_{\mathrm{cs}} V_{\mathrm{us}}^{*}\right]}{\lambda^{2}} \frac{P}{S} \sin \delta_{\mathrm{str}} \\
& \sim A^{2} \eta \lambda^{4} \frac{P}{S} \sin \delta_{\mathrm{str}} \leq 10^{-3},
\end{aligned}
$$

where $\lambda, A$ and $\eta$ are the Wolfenstein parameters of the CKM matrix.

\section{Theory of $\bar{D}^{0}-D^{0}$ Mixing in the Standard Model}

In the following, we give an up-to-date and reasonably thorough review of attempts to theoretically determine $\left|\Delta M_{D}\right|$ and $\left|\Delta \Gamma_{D}\right|$.

\subsection{Short Distance ('Box') Contribution}

Analytic formulae for the box contribytions to $\left|\Delta M_{D}\right|$ and $\left|\Delta \Gamma_{D}\right|$ first appeared in the literature over a decade ago, 21 and were given in approximate form for $D^{0}-\bar{D}^{0}$ mixing shortly thereafter. 22 The precise formulae are

$$
\begin{aligned}
\Delta M_{D}^{\text {box }} & =\frac{2}{3 \pi^{2}} X_{D} \frac{\left(m_{s}^{2}-m_{d}^{2}\right)^{2}}{m_{c}^{2}}\left[1-\frac{5}{4} \frac{B_{D}^{\prime}}{B_{D}} \frac{M_{D}^{2}}{\left(m_{c}+m_{u}\right)^{2}}\right] \\
\Delta \Gamma_{D}^{\text {box }} & =\frac{4}{3 \pi} X_{D} \frac{\left(m_{s}^{2}-m_{d}^{2}\right)^{2}}{m_{c}^{2}} \frac{m_{s}^{2}+m_{d}^{2}}{m_{c}^{2}}\left[1-\frac{5}{2} \frac{B_{D}^{\prime}}{B_{D}} \frac{M_{D}^{2}}{\left(m_{c}+m_{u}\right)^{2}}\right]
\end{aligned}
$$


Table 6: Box Contributions to $\Delta M_{D}$ and $\Delta \Gamma_{D}$.

\begin{tabular}{c|c|c|c}
$m_{c}(\mathrm{GeV})$ & $\Delta M_{D}(\mathrm{GeV})$ & $\Delta \Gamma_{D}(\mathrm{GeV})$ & $r_{\text {box }}$ \\
\hline \hline 1.30 & $-0.191 \times 10^{-16}$ & $-0.752 \times 10^{-17}$ & 0.395 \\
1.32 & $-0.176 \times 10^{-16}$ & $-0.681 \times 10^{-17}$ & 0.388 \\
1.34 & $-0.162 \times 10^{-16}$ & $-0.618 \times 10^{-17}$ & 0.381 \\
1.36 & $-0.150 \times 10^{-16}$ & $-0.561 \times 10^{-17}$ & 0.375 \\
1.38 & $-0.138 \times 10^{-16}$ & $-0.510 \times 10^{-17}$ & 0.369 \\
\hline \hline
\end{tabular}

where the $b$-quark contribution has been neglected, $X_{D}$ is given by $X_{D} \equiv$ $\xi_{d}^{2} B_{D} G_{F}^{2} M_{D} F_{D}^{2}$, with $\xi_{d} \equiv V_{c d}^{*} V_{u d}$ and no QCD corrections are yet included. The squared GIM suppression factors $\left(m_{s}^{2}-m_{d}^{2}\right)^{2} / m_{c}^{2}$ indicate the presence of two $|\Delta C|=1$ transitions and there is an additional suppression factor $\left(m_{s}^{2}+\right.$ $\left.m_{d}^{2}\right) / m_{c}^{2}$ for $\Delta \Gamma_{D}$. Until recently, the 'B-parameters' $B_{D}$ and $B_{D}^{\prime}$ represented a major source of numerical uncertainty, even as to the sign of the effect. However, a very recent lattice determination 23 of both the B-parameters has alleviated this source of numerical ambiguity, leaving the value of $m_{c}$ as perhaps the least well-determined quantity. We display the $m_{c}$-dependence of the box contributions to $\Delta M_{D}, \Delta \Gamma_{D}$ and $r_{\text {box }} \equiv \Delta \Gamma_{D} / \Delta M_{D}$ in Table 6 .

\subsection{Heavy Quark Effective Theory Analysis}

The effect of QCD radiative corrections on the box contribution has been studied in the context of the heavy quark effective theory (HQET). Georgi gave the original formulation of HQET to $D^{0}-\bar{D}^{0}$ mixing, 44 and analysis of the QCD effects appeared soon thereafter.25 HQET represents the most natural means for computing QCD radiative corrections for heavy quark processes, as otherwise uncontrollable logarithmic factors are avoided. There are several steps in this approach: (i) obtain an effective hamiltonian in terms of local operators (OPE), (ii) run the energy scale (RG equations) from $\mu \simeq M_{\mathrm{W}}$ down to the value of interest ( $\mu \simeq m_{c}$ in our case) and (iii) obtain accurate values for the matrix elements of the local operators. For application to $D^{0}-\bar{D}^{0}$ mixing, operators of dimension four, six and eight were considered, and the maximum effect obtained was estimated to be $\left|\Delta M_{D}\right| \leq 3.5 \times 10^{-17} \mathrm{GeV}$. 25 The main uncertainites here involve convergence of the OPE (i.e. is the $c$-quark really heavy?) and evaluation of the multi-quark matrix elements (effects of nonperturbative physics). 
Table 7: One-particle Contributions to $\Delta m_{D}$.

\begin{tabular}{c|c||c|c} 
Mode & $\Delta m_{D}(\mathrm{GeV})$ & $\theta_{P}(\mathrm{deg})$ & $\Delta m_{D}(\mathrm{GeV})$ \\
\hline \hline$K$ & $0.1345 \cdot 10^{-15}$ & -13 & $0.4127 \cdot 10^{-16}$ \\
$B_{d}$ & $-0.1830 \cdot 10^{-17}$ & -14 & $0.3948 \cdot 10^{-16}$ \\
$B_{s}$ & $0.2407 \cdot 10^{-17}$ & -15 & $0.3757 \cdot 10^{-16}$ \\
$\pi$ & $-0.1545 \cdot 10^{-17}$ & -16 & $0.3554 \cdot 10^{-16}$ \\
$\eta_{B}$ & $0.7138 \cdot 10^{-21}$ & -17 & $0.3339 \cdot 10^{-16}$ \\
$\eta$ & $-0.7227 \cdot 10^{-16}$ & -18 & $0.3112 \cdot 10^{-16}$ \\
$\eta^{\prime}$ & $-0.3250 \cdot 10^{-16}$ & -19 & $0.2875 \cdot 10^{-16}$ \\
Total & $0.2875 \cdot 10^{-16}$ & -20 & $0.2627 \cdot 10^{-16}$ \\
\hline \hline
\end{tabular}

\subsection{Dipenguin Contribution}

The box contribution is not the only short distance contribution. It has long been understood that the effect of twopenguin operators (dipenguin) can also occur as a four-quark local operator.26 This effect has been studied for both the $K^{0}-\bar{K}^{0}$ and $B^{0}-\bar{B}^{0}$ mixings, but until recently not for $D^{0}-\bar{D}^{0}$ mixing. This gap in the literature has been filled by a very recent calculation of Petrov who finds 27

$$
\left|\Delta M_{D}^{\mathrm{di}-\mathrm{pen}}\right| \leq 10^{-17} \mathrm{GeV} .
$$

Thus, the dipenguin does not lead to a surprisingly large contribution.

\subsection{Long Distance ('Dispersive') Contributions}

It could happen at the relatively light charm mass scale that significant corrections to the short-distance box contribution exist. It is difficult to obtain an accurate determination of this long distance component, but nonetheless instructive to study the various categories.

\section{Single-particle Sector}

Although the analysis of the single particle sector was carried out some time ago, 28 this represents its first published appearance. Individual contributions are displayed in Table 7 for a specific choice $\theta_{P}=-19^{\circ}$ of the $\eta-\eta^{\prime}$ mixing angle, and the dependence on $\theta_{P}$ is given in the two rightmost columns. 


\section{Multi-particle Sector}

Historically, the first of the long distance pmponents to be studied was the two-particle charged-pseudoscalar sector. 29.30 The motivation was then (and remains to this day) quite clear - the large $S U(3)$ breaking evidenced in $D$ decays. One example of this concerns the ratio $\Gamma_{D^{0} \rightarrow K^{+} K^{-}} / \Gamma_{D^{0} \rightarrow \pi^{+} \pi^{-}} \simeq 3$, which is unity in the $S U(3)$ limit. Chau and Chang have analyzed several related decays and find the large breaking to be an accumulation of a number relatively minor effects whose ultimate impact is large. 31

In the original quantitative study of the long distance two-particle sector, 30 an approximate correspondence $\left|\Delta M_{\mathbb{R}}^{\text {disp }} / \Delta \Gamma_{D}\right| \sim 0.2$ appeared natural. If we combine this with a recent analysis ${ }^{2}$ which estimates $\Delta \Gamma_{D} / \Gamma_{D} \simeq 10^{-3}$ via an explicit sum over two-particle $P P, V P, S P$ modes, we obtain the following order-of-magnitude result,

$$
\begin{aligned}
\left|\Delta M_{D}^{\text {disp }}\right| & \sim 0.2 \Delta \Gamma_{D} \quad \text { (heuristic assumption) } \\
& =0.2 \frac{\Delta \Gamma_{D}}{\Gamma_{D}} \Gamma_{D} \\
& \sim 0.2 \times 10^{-3} \times 10^{-12} \mathrm{GeV} \sim \mathcal{O}\left(10^{-16} \mathrm{GeV}\right)
\end{aligned}
$$

Although this order-of-magnitude estimate is far from rigorous, it represents a reasonable bound on the magnitude of the dispersive component.

\section{New Physics}

Although a variety of important SM tests remain to be carried out, increasing attention is being given to probes of new physics. In the following, we recall some recent literature regarding the time-dependent decay rate $\Gamma_{D^{0}(t) \rightarrow f}$ and then catalog a number of potential new physics contributions to $\Delta M_{D}$.

\subsection{Time Dependence in $\bar{D}^{0}-D^{0}$ Mixing}

Several papers have pointed out the potential importance of the interference term ( $c f$ the $Y$-term in Eq. (4)) for studies of mixing in the $D$ sector. First, Wolfenstein noted that detection of mixing at current levels of sensitivity would indicate new physics, and that in the absence of FSI, the effect would involve CPV. 32 Papers by Blaylock, Seiden \& Nir 19 and by Browder \& Pakvasa 33 provided a more detailed look at new physics possibilities. The latter also stressed that the term $Y$ would survive (i) for zero CPV in the combination $\Gamma_{D^{0}(t)+\bar{D}^{0}(t)}$ and (ii) for zero FSI in the combination $\Gamma_{D^{0}(t)-\bar{D}^{0}(t)}$. A summary of CPV contributions to $D^{0}-\bar{D}^{0}$ mixing from a variety of new physics scenarios exists in preprint version. 3 . 


\subsection{Possible Contributions to $\bar{D}^{0}-D^{0}$ Mixing}

A propos of new physics contributions to a given system, there is by now a well-developed path to follow:

- Pick some Model-X of new physics, identifying its degrees of freedom and the extent of its parameter space,

- Calculate its effect on a given observable,

- Perform a phenomenological analysis and decide whether

(a) Model-X contribution unobservable?

(b) Model-X parameter space constrained?

(c) Model-X ruled out?

Table 8 displays a rich spectrum of new physics possibilities for contributing to $D^{0}-\bar{D}^{0}$ mixing. Indeed, all the models typically discussed in the literature are available. We display some of the mechanisms for inducing $D^{0}-\bar{D}^{0}$ mixing in Figure 1 .

The remainder of the procedure - to calculate the effects over the available parameter space - is reviewed ably by Hewett 35 and by Burdman, 20 who also refer to the original literature. We simply note here that:

(i) Although the HERA-motivated interest in leptoquarks is quite recent, there already exist interesting phenomenological studies of such particles, including their effect on $D^{0}-\bar{D}^{0} .36$

(ii) In the flavor-changing neutral Higgs scenario, it has recently been pointed out that a reasonably modest improvement in the bound on $D^{0}-\bar{D}^{0}$ mixing will make this test competitive with mixing constraints in the kaon and $B_{d}$ systems. 37

(iii) The tight experimental bounds on FCNC processes place severe restrictions in supersymmetric models on the structure of sfermion mass matrices. Several approaches ('flavor universality', 'alignment', 'gauge mediation') are available for dealing with this problem. We note a recent 'mass insertion approximation' analysis of FCNC constraints which is relatively model independent and includes a study of $D^{0}-\bar{D}^{0}$ mixing. 38

\section{Summary}

We have argued for using B-factory capabilities to carry out charm physics experiments in the three areas of rare decay searches, CPV asymmetry searches and improvement of the bound on $D^{0}-\bar{D}^{0}$ mixing. Each of these has greater 


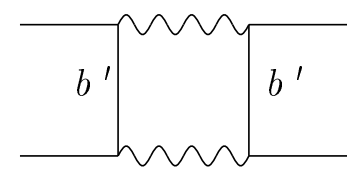

(a)

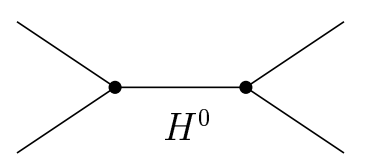

$(c)$

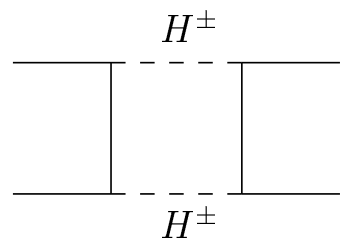

(b)

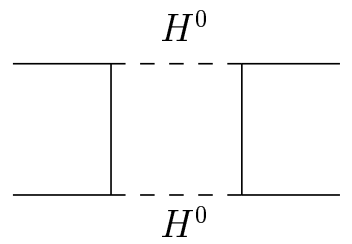

$(d)$

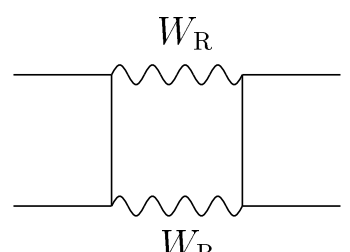

$(e)$

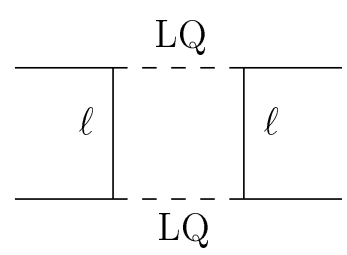

$(f)$

\begin{tabular}{|c|c|}
\hline & \\
\hline $\begin{array}{l}\tilde{q}_{1} \\
\tilde{q}_{2}\end{array}$ & $\begin{array}{c}\tilde{q}_{2} \\
\tilde{q}_{1}\end{array}$ \\
\hline
\end{tabular}

$(g)$

Figure 1: Effects of new physics on $\bar{D}^{0} D^{0}$ Mixing: (a) extra $Q=-1 / 3$ quark $b^{\prime}$, (b) charged higgs scalars $H^{ \pm}$, (c-d) tree, box contributions of flavor-changing neutral higgs scalars $H^{0}$, (e) left-right symmetric $W$-boson $W_{R}$, (f) leptoquark LQ, (g) gluino $\tilde{g}$ and squarks $\tilde{q}_{1,2}$. 
Table 8: New Physics Possibilities.

\begin{tabular}{l|l} 
New Physics & Comment \\
\hline \hline Extra quarks & $\begin{array}{l}4^{t h} \text { family weak-isodoublet }\left(t^{\prime}, b^{\prime}\right) \\
\text { singlets }(Q=-1 / 3 \text { or } Q=+2 / 3) \\
\text { charged higgs } H^{ \pm} \\
\text {neutral }(\mathrm{FC}) \text { higgs }\left(H^{0}\right) \\
\text { left-right symmetry }\left(W_{R}\right)\end{array}$ \\
Extra W-bosons & Reptoquarks \\
Sparticles & $\begin{array}{l}\text { R-parity conserving } \\
\text { techniparticles }\end{array}$ \\
Compositeness & \\
Family symmetry & \\
\hline \hline
\end{tabular}

intrinsic interest than more conservative choices. To support this view, an up-to-date summary of these topics has been provided, and several new results (particularly in the discussion of $D^{0}-\bar{D}^{0}$ mixing) have been presented. Additional studies are underway and theoretical advances in the near term are anticipated. We end with the reminder that there are advantages to doing $D^{0}-\bar{D}^{0}$ mixing searches at an asymmetric $B$-factory, particularly using a hadronic tag and a semileptonic mixing signal. 39

\section{Acknowledgments}

Research by the UMass theory group is supported by the National Science Foundation. The input of my collaborators Gustavo Burdman, JoAnne Hewett and Sandip Pakvasa and of Mark Windoloski is gratefully acknowledged.

\section{References}

1. Part. Data Group, Phys. Rev. D 54, 1 (1996).

2. CLEO collaboration, Cornell Univ. preprint CLNS 96/1449 (Dec. 1996).

3. T.E. Browder, K. Honscheid and D. Pedrini, Ann. Rev. Nucl. Part. Sci. 46, 395 (1997).

4. F. Buccella et al, Phys. Rev., D51 (1995) 3478; F. Buccella, M. Lusignoli and A. Pugliese, Phys. Lett., B379 (1996) 249.

5. D. Cinabro et al (CLEO collaboration), Phys. Rev. Lett., 72 (1994) 1406. 
6. E.M. Aitala et al (E791 collaboration), submitted to Phys. Rev. D, hep-ex/9608018.

7. P.L. Frabetti et al (E687 collaboration), Phys. Lett., B359 (1995) 403.

8. E.M. Aitala et al (E791 collaboration), FERMILAB-PUB-97-053-E (March 1997).

9. E.M. Aitala et al (E791 collaboration), Phys. Rev. Lett., 76 (1996) 364.

10. T. Alexopoulos et al (E771 collaboration), Phys. Rev. Lett., 77 (1996) 2380 .

11. A. Freyberger et al (CLEO collaboration), Phys. Rev. Lett., 77 (1996) 810.

12. For example, see S. Pakvasa, talk delivered at FCNC97 Conference, hep$\mathrm{ph} / 9705397$.

13. A theoretical survey of long-range vs. short-range effects for various rare $D$-meson decay modes is in preparation: G. Burdman, E. Golowich, J. Hewett and S. Pakvasa (unpublished).

14. G. Burdman, E. Golowich, J. Hewett and S. Pakvasa, Phys. Rev. D52 6383 (1995).

15. C. Greub, T. Hurth, M. Misiak and D. Wyler, Phys. Lett. B 382, 415 (1996).

16. A. Khodjamirian, G. Stoll and D. Wyler, Phys. Lett., B358 (1995) 129.

17. P. Singer and D-X Zhang, Phys. Rev. D55 (1997) R1127.

18. T. Liu, Princeton University preprint HEP/95-6 (June 1995), hep$\mathrm{ph} / 9608415$.

19. E.g., see G. Blaylock, A. Seiden and Y. Nir, Phys. Lett., B355 (1995) 555 .

20. G. Burdman, Potential for Discoveries in Charm Meson Physics, presented at Workshop on the Tau/Charm Factory, Argonne (6/95), hep$\mathrm{ph} / 9508349$.

21. For example, see J.S. Hagelin, Nucl. Phys. B193 (1981) 123; A.J. Buras, M. Jamin and P.H. Weisz, Nucl. Phys. B347 (1990) 491.

22. A. Datta and M.Khambakhar, Zeit. Phys., C27 (1985) 515.

23. R. Gupta, T. Bhattacharya and S. Sharpe, Phys. Rev. D 55, 4036 (1997).

24. H. Georgi, Phys. Lett., B297 (1992) 353.

25. T. Ohl et al, Nucl. Phys. B403 (1993) 605.

26. J. Donoghue, E. Golowich and G. Valencia, Phys. Rev. D33 (1986) 1387.

27. A. Petrov, Phys. Rev. (in press), hep-ph/9703335.

28. G. Burdman, E. Golowich, J. Hewett and S. Pakvasa (unpublished).

29. L. Wolfenstein, Phys. Lett., B164 (1985) 170. 
30. J. Donoghue, E. Golowich, B. Holstein and J. Trampetic, Phys. Rev., D33 (1986) 179.

31. L-L Chau and H-Y Cheng, Phys. Lett. B333 (1994) 514.

32. L. Wolfenstein, Phys. Rev. Lett., 75 (1995) 2460.

33. T.E. Browder and S. Pakvasa, Phys. Lett., B383 (1996) 475.

34. G. Burdman, E. Golowich, J. Hewett and S. Pakvasa, SLAC preprint SLAC-PUB-95-7076 (Dec. 1995).

35. J. Hewett, Stanford preprint SLAC-PUB-95-6821 (April 1995).

36. For example, see S. Davidson, D. Bailey and B.A. Campbell, Z. Phys. C61 (1994) 613.

37. D. Atwood, L. Reina and A. Soni, Phys. Rev. D55 (1997) 3156.

38. F. Gabbiani, E. Gabrielli, A. Masiero and L. Silvestrini, Nucl. Phys. B477 (1996) 321.

39. G. Blaylock, private communication. 